\title{
Vaginal progesterone for prevention of preterm birth in asymptomatic high-risk women with a normal cervical length: a systematic review and meta-analysis protocol
}

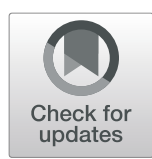

Kimberley P. Williams ${ }^{1}$, Liam McAuliffe ${ }^{1}$, Rosanna Diacci ${ }^{1}$, Anne-Marie Aubin ${ }^{1}$, Ashad Issah ${ }^{1}$, Carol Wang ${ }^{1,2}$, Jason Phung ${ }^{1,2,3^{*}}$ (D) and Craig E. Pennell ${ }^{1,2,3}$

\begin{abstract}
Background: Preterm birth (PTB) is estimated to affect 14.9 million babies globally every year. Global rates of PTB continue to increase from 9.8 to $10.6 \%$ over a 15-year period from 2000 to 2014. Vaginal progesterone is commonly used by clinicians as a prevention strategy, with recent evidence affirming the benefit of vaginal (micronised) progesterone to prevent PTB in women with a shortened cervix $(<25 \mathrm{~mm})$. Given the low incidence of a short cervix at mid-gestation in high-risk populations further evidence is required. The objective of this review is to determine if vaginal progesterone reduces spontaneous preterm birth (sPTB) before 37 weeks in asymptomatic high-risk women with a singleton pregnancy with a normal mid-gestation cervical length.

Methods: Studies will be sourced from MEDLINE, Embase and Cochrane Register of Trials (CENTRAL) from their inception onwards with the search terms 'progesterone' and 'preterm birth'. Studies will be screened and included if they assess vaginal progesterone compared to placebo in women with a normal cervical length. The primary outcome will be sPTB $<37$ weeks, with secondary outcomes of sPTB $<34$ weeks.

Two independent reviewers will conduct study screening at abstract and full text level, data extraction and risk of bias assessment with disagreements resolved by an experienced researcher. The Mantel-Haenszel statistical method and random effects analysis model will be used to produce treatment effect odds ratios and corresponding $95 \%$ confidence intervals.
\end{abstract}

Discussion: This review will assess the current body of evidence and provide clarity regarding the potential benefits and best practice of use of vaginal progesterone in asymptomatic women with high-risk singleton pregnancies and normal cervical length.

Trial registration: PROSPERO CRD42020152051

Keywords: Progesterone, Preterm birth, Cervical

\footnotetext{
* Correspondence: Jason.Phung@health.nsw.gov.au

'School of Medicine and Public Health, The University of Newcastle, Callaghan, New South Wales, Australia

${ }^{2}$ Mothers and Babies Research Centre, Hunter Medical Research Institute,

New Lambton Heights, New South Wales, Australia

Full list of author information is available at the end of the article
}

(c) The Author(s). 2021 Open Access This article is licensed under a Creative Commons Attribution 4.0 International License, which permits use, sharing, adaptation, distribution and reproduction in any medium or format, as long as you give appropriate credit to the original author(s) and the source, provide a link to the Creative Commons licence, and indicate if changes were made. The images or other third party material in this article are included in the article's Creative Commons licence, unless indicated otherwise in a credit line to the material. If material is not included in the article's Creative Commons licence and your intended use is not permitted by statutory regulation or exceeds the permitted use, you will need to obtain permission directly from the copyright holder. To view a copy of this licence, visit http://creativecommons.org/licenses/by/4.0/ The Creative Commons Public Domain Dedication waiver (http://creativecommons.org/publicdomain/zero/1.0/) applies to the data made available in this article, unless otherwise stated in a credit line to the data. 


\section{Introduction}

Preterm birth (PTB) is estimated to affect 14.9 million babies worldwide every year [1], with a higher burden in low- and middle-income countries [2]. This has generated a geographic divide with several European countries such as Finland and Sweden having the lowest PTB rates $<6 \%$, whilst low income countries such as Malawi in Africa, have the highest PTB rate of $18.1 \%$ [3]. Similarly, estimated global PTB rates have increased from 9.8\% in 2000 to $10.6 \%$ in 2014 [2]. Despite approximately $80 \%$ of these preterm births occurring in disadvantaged countries in sub-Saharan Africa and Asia, data suggests that rates of PTB are also steadily increasing in high-income countries [2]. This is a worrying trend as PTB remains the leading cause of death for children under the age of five [4]. Furthermore, it increases the risk of short-term complications including infant respiratory distress syndrome and intraventricular haemorrhage, as well as life-long impacts including increased risk of neurodevelopmental delay and adult non-communicable diseases [5].

A number of interventions have been shown to significantly decrease the rate of spontaneous preterm birth (sPTB) including specialist antenatal clinics for women at high risk of PTB [6]; cervical cerclage for women with a previous SPTB and/or a short cervix $(<25 \mathrm{~mm})[7,8]$ and vaginal progesterone for women with cervical length $<25 \mathrm{~mm}$ [9-13]. Progesterone decreases uterine contractility through inhibition of production of prostaglandins in the myometrium [14] and has been shown to be important in maintaining a pregnancy until term [15].

A recent individual patient data (IPD) meta-analysis affirmed the benefit of vaginal progesterone in prevention of sPTB in women with a short cervix $(<25 \mathrm{~mm})$ with a $20 \%$ reduction in sPTB before 36 weeks, and a greater reduction of $35 \%$ before 34 weeks [8]. This IPD suggested that vaginal progesterone is still beneficial even in nulliparous women with only a short cervix as a risk factor [8]. As only 5.8-7.3\% of high-risk women [9, $10]$, and $0.45-1.68 \%$ in unselected populations [11-13] have a short cervix at mid gestation, the number of women who benefit from progesterone is relatively low. It is currently unclear whether vaginal progesterone would be of benefit to the other high-risk women with a normal mid-gestation cervical length (> $25 \mathrm{~mm}$ ).

Whilst there is a substantial body of research into progesterone and PTB prevention, current literature focuses on women with a short cervix and fails to address whether vaginal progesterone would benefit high-risk women specifically with a normal mid-gestation cervical length (> $25 \mathrm{~mm}$ ). Many studies assessing the effectiveness of vaginal progesterone in based on risk factors alone did not concurrently measure the cervical length and therefore we cannot be confident that these populations did not contain patients with a short cervix [16].
The most recent systematic review assessing the role of progesterone in PTB reduction [17] has done so by combining studies where progesterone was administered orally, vaginally and intramuscularly (17-OHP). There is evidence to suggest that the mechanism of action differ [18] and therefore should be studied separately. This is supported by the fact that vaginal progesterone and IM 17-OHP appear to be effective in different populations $[19,20]$. Whilst several publications comparing the route of administration exist [21], this is beyond the scope of this review.

Recommendations currently exist for the administration of vaginal progesterone for women with a short cervix and therefore many centres in high resource countries are able to offer cervical length screening as routine care. We know from prior publications that the rate of a short sonographic cervix is low [10]. Therefore, this proposed systematic review will answer the question does vaginal progesterone reduce spontaneous PTB in asymptomatic high-risk women with a singleton pregnancy with a normal mid-gestation cervical length?

\section{Methods}

The present protocol has been registered within the PROSPERO database (registration number CRD42020152051) and is being reported in accordance with the reporting guidance provided in the Preferred Reporting Items for Systematic Reviews and Meta-Analyses Protocols (PRISMA-P) statement (see checklist in Additional file 1).

\section{Information sources and search strategy}

The search strategy will be developed focusing on identifying the relevant intervention with no populationrelated keywords. The primary source of literature will be a structured search of the electronic databases MEDL INE, EMBASE and Cochrane Register of Trials (CENT RAL) from their date of inception onwards. The secondary source of literature will be a search of the grey literature, including Google Scholar. We will perform hand-searching of the reference lists of included studies, relevant reviews, national clinical practice guidelines or other relevant documents. We will contact content experts and authors who are prolific in the field. The literature searches will be designed and conducted by the review team with an experienced health information specialist. The search will include the following search terms: preterm birth OR premature birth AND progesterone. We will place no restriction on the length of study follow-up time, or on country, year or language of publication. However, the search will be limited to studies on humans. Medical subject headings (MeSH) will be used when relevant. Where appropriate, the authors of studies will be contacted to provide additional 
information. A draft search strategy for MEDLINE is provided in Additional file 2.

\section{Eligibility criteria}

The eligibility of studies included will be based on inclusion and exclusion criteria applied to the domains of participant, exposure, comparator, study type and outcome.

\section{Participants}

This review will consider all studies that include asymptomatic pregnant women being treated with vaginal progesterone. We will exclude studies that include women with a short cervix or who were symptomatic. Studies that exclusively studied women with multi-gestation will be excluded.

\section{Intervention and comparators}

Studies comparing vaginal progesterone either compared to placebo or no treatment will be included. Studies that involved other methods of progesterone administration such as intramuscular injection will be excluded.

Vaginal progesterone is available as a gel, suppository or pessary [14]. It is the most bioavailable form of progesterone for uterine and cervical effects with the fewest side effects. Its micronisation decreases particle size and increases surface area. This results in improved absorption with less metabolic and vascular side effects [22]. The vaginal route also allows rapid absorption and avoids first pass hepatic metabolism, resulting in high bioavailability in the uterus [23].

\section{Outcomes}

The primary outcome is sPTB before $<37$ weeks gestation.

Preterm birth will be defined as live or stillbirth with a gestational age between 20 and 37 weeks.

The secondary outcome is sPTB before $<34$ weeks gestation.

\section{Types of studies}

The review will include randomised controlled trials. All included papers must include vaginal progesterone compared to either placebo or no treatment. Those studies which also present a control group will be included.

We will place no restriction on the country, year and language of publication or the length of study follow-up. Included studies will be limited to human trials.

\section{Selection and data collection process Study selection}

Titles and abstracts identified through all sources will be downloaded to Endnote [24] and duplicates will be removed. Studies will then be screened using the specified eligibility criteria above and studies that do not meet the criteria will be excluded. Full texts of remaining studies will be screened before undergoing critical appraisal and data extraction. All levels of screening will be conducted by two independent reviewers and any disputes between reviewers will be resolved by an independent moderator. None of these reviewers will be blinded to titles, authors, journals or institutions.

\section{Data management}

The search will be uploaded to an Endnote [24] library, which allows collaboration between multiple reviewers during the study selection process.

\section{Data collection}

Two reviewers will extract data through Endnote [24] using a standardised electronic data extraction sheet. Any discrepancies will be moderated by a third senior research reviewer. Once extracted, upon reviewer agreement, data will be transferred into Review Manager version 5.3 data-analysis software [25].

The following data will be extracted:

- Study characteristics: authors; publication date; study design; country of study; sample size; confounding factors of participants; publication status; trial size; funding and risk of bias information.

- Intervention characteristics: type of intervention used; reason for intervention; patient characteristics (maternal age, gravity, parity, cervical length) and any co-interventions received.

- Outcomes: maternal, foetal and neonatal outcome data and definitions of each of the outcomes as described below.

\section{Risk of bias in individual studies}

Risk of bias for each paper will be assessed using the Joanna Briggs Institute critical appraisal method for randomised control trials [26]. Studies which are deemed to have not addressed the possibility of bias in the design, conduct or analysis will then be excluded.

\section{Confidence of cumulative evidence}

To assess the strength of the body of evidence, we will utilise the Cochrane GRADE tool [27]. Evidence will be assessed in terms of risk of bias, consistency, directness, precision and publication bias. With regard to GRADE, quality will be assessed as being one of 4 grades: (i) high-we are very confident that the true effect is close to that of the estimate of the effect; (ii) moderate-we are moderately confident in the effect estimate, and the true effect is likely to be close to the estimate of the effect, but there is a possibility that it is substantially 
different; (iii) low-our confidence in the effect estimate is limited, and the true effect may be substantially different from the estimate of the effect; and (iv) very lowwe have very little confidence in the effect estimate, and the true effect is likely to be substantially different from the estimate of effect. Two independent reviewers will conduct the assessment, with discrepancies resolved through discussion and consensus between the two reviewers, or consultation with a third reviewer.

Review Manager version 5.3 [25] will be used to compute graphic representations of potential bias within and across studies.

\section{Data synthesis}

Meta-analysis will be constructed by pooling studies using Covidence and RevMan 5.3. In order for synthesis, studies must report outcomes appropriately (i.e. $<37$ for the primary outcome) or provide sufficient information to calculate the outcome(s) of interest. We will synthesise published data from the original manuscripts, appendices or any data provided by authors upon request. We will only synthesise data based on the primary research question of vaginal progesterone vs. placebo and therefore will accept varying doses provided they have the correct route of administration. Given the broad nature of the 'at risk' population, we expect there to be significant heterogeneity within these studies and will test for this using statistical methods such as Chi2 and $\mathrm{I}^{2}$ statistics.

If feasible and appropriate, outcome data will be used to perform random effects meta-analyses because of anticipated heterogeneity. The random effects model assumes the study level effect estimates follow a normal distribution, considering both within-study and between-study variation.

Dichotomous outcomes will be assessed and reported using risk ratios and 95\% confidence intervals will be used. For continuous outcomes, trial data will be reported using mean differences (MDs) or standardised mean differences (SMDs) and 95\% continuous outcome variables. Where meta-analysis is not possible, alternative synthesis methods, including summarising effect estimates, will be used as recommended by the Cochrane Handbook for Systematic Reviews of Interventions [28].

\section{Missing data}

For studies which present missing data, we will attempt to contact authors. However, if this is not possible, we will conduct sensitivity analysis which will exclude trials with $>30 \%$ missing data.

\section{Meta-bias(es)}

Meta-biases will be assessed by examining funnel plots for symmetry and Egger's test may be used for assessing publication bias.

\section{Additional analyses}

Sensitivity analysis will be conducted on the primary outcome for sPTB < 37weeks gestation by removing studies which are judged to have an overall high risk of bias, allowing us to examine their impact on the effect estimate of the primary outcome. Data will be extracted from each individual study regarding baseline maternal characteristics (age, gestation, risk of bias, setting and geographical region) and neonatal outcomes where individual patient data is available and pertinent sub-group analyses will be conducted if there are vast differences in baseline characteristics.

\section{Discussion}

This systematic review and meta-analysis aims to determine the effectiveness of vaginal progesterone for prevention of PTB in asymptomatic high-risk women with a normal cervical length. It is hoped this paper will synthesise multiple recent large scale trials to provide valuable therapeutic information to specialists in their clinical decisions for women at risk of PTB. It is hoped women at high risk of obstetric complications, their families and the wider community will benefit from these findings. The results of this paper will help to inform guidelines and reduce the short- and long-term negative health outcomes of preterm birth.

Whilst we do not anticipate any major operational issues in the conduction of this review, an issue we foresee as possible would be if the included studies do not have individual patient data. If this is the case, we plan to overcome this by contacting the authors of the individual studies about whether individual patient data is available.

Firstly, in terms of study selection, there may not be enough studies in the area to yield significant results and/or these studies may be of poor quality. Secondly, studies may not report individual patient data and may only include summary of results which limits the analyses that can be reached in our meta-analysis.

Should there be any major changes that the existing protocol that significantly affect the scope of the investigation, safety of the subjects or quality of the study, a protocol amendment will be submitted.

\section{Plan for dissemination}

We plan for this systematic review and meta-analysis to be disseminated through peer-reviewed publication.

\section{Abbreviations}

$\mathrm{Cl}$ : Confidence interval; CINAHL: Cumulative Index of Nursing and Allied Health Literature; GRADE: Grading of Recommendations, Assessment, Development and Evaluations; IPD: Individual patient data; MD: Mean difference; MeSH: Medical subject headings; P value: Probability value; PRIS MA-P: Preferred Reporting Items or Systematic Reviews and Meta-Analyses Protocol; PPROM: Preterm premature rupture of membranes;

PROSPERO: International Prospective Register of Systematic Reviews; 
RCT: Randomised control trial; RDS: Respiratory distress syndrome; RevMan: Review Manager 5.3; ROBINS I: Risk of Bias in Non-Randomised Studies of Interventions; ROBINS II: Risk of Bias in Randomised Studies of Interventions; RR: Risk ratio; SPTB: Spontaneous preterm birth; SMD: Standardised mean difference

\section{Supplementary Information}

The online version contains supplementary material available at https://doi. org/10.1186/s13643-021-01702-9.

Additional file 1. PRISMA-P Checklist

Additional file 2. Search Strategy

\section{Acknowledgements}

Not applicable.

\section{Authors' contributions}

K.P.W. and L.M. led the writing of this manuscript with input from R.D., A.I. and A-M.A.

J.P. and C.E.P. conceived and designed the paper. C.W. provided guidance on the statistical plan, and J.P. and C.E.P. provided expertise relating to obstetric care. The authors read and approved the final manuscript.

\section{Funding}

J.P is funded by the Hunter New England Local Health District, Clinical Health Service Research Fellowship Scheme (CHSRFS). The funder played no role in the development of the protocol.

\section{Availability of data and materials}

Not applicable.

\section{Declarations}

\section{Ethics approval and consent to participate}

Not applicable.

\section{Consent for publication}

Not applicable.

\section{Competing interests}

J.P. is supported by the Hunter New England Health Local Health District Clinical and Health Service Research Fellowship Scheme. The authors declare that they have no other competing interests.

\section{Author details}

${ }^{1}$ School of Medicine and Public Health, The University of Newcastle, Callaghan, New South Wales, Australia. '2Mothers and Babies Research Centre, Hunter Medical Research Institute, New Lambton Heights, New South Wales, Australia. ${ }^{3}$ Maternity and Gynaecology, John Hunter Hospital, New Lambton Heights, New South Wales, Australia.

\section{Received: 18 September 2020 Accepted: 13 May 2021}

Published online: 21 May 2021

\section{References}

1. Blencowe $H$, Cousens S, Oestergaard MZ, Chou D, Moller A-B, Narwal R, et al. National, regional, and worldwide estimates of preterm birth rates in the year 2010 with time trends since 1990 for selected countries: a systematic analysis and implications. Lancet. 2012;379(9832):2162-72. https://doi.org/10.1016/S0140-6736(12)60820-4.

2. Chawanpaiboon S, Vogel JP, Moller A-B, Lumbiganon P, Petzold M, Hogan $D$, et al. Global, regional, and national estimates of levels of preterm birth in 2014: a systematic review and modelling analysis. Lancet Glob Health. 2019; 7(1):e37-46. https://doi.org/10.1016/S2214-109X(18)30451-0

3. WHO. recommended definitions terminology format for statistical tables related to the perinatal period use of a new certificate for cause of perinatal deaths. Modifications recommended by FIGO as amended October 14 1976. Acta Obstet Gynecol Scand. 1977;56:247-53.
4. Goldenberg RL, Culhane JF, lams JD, Romero R. Epidemiology and causes of preterm birth. The Lancet. 2008;371(9606):75-84. https://doi.org/10.1016/ S0140-6736(08)60074-4.

5. Ward RM, Beachy JC. Neonatal complications following preterm birth. Br J Obstet Gynaecol. 2003;110:8-16. https://doi.org/10.1016/S1470-032 8(03)00012-0.

6. Malouf R, Redshaw M. Specialist antenatal clinics for women at high risk of preterm birth: a systematic review of qualitative and quantitative research. BMC Pregnancy Childbirth. 2017;17(1):51. https://doi.org/10.1186/s12884-01 7-1232-9.

7. Berghella V, Mackeen AD. Cervical length screening with ultrasoundindicated cerclage compared with history-indicated cerclage for prevention of preterm birth: a meta-analysis. Obstet Gynecol. 2011;118(1):148-55. https://doi.org/10.1097/AOG.0b013e31821fd5b0

8. Souza RT, Costa ML, Mayrink J, Feitosa FE, Rocha Filho EA, Leite DF, et al. Clinical and epidemiological factors associated with spontaneous preterm birth: a multicentre cohort of low risk nulliparous women. Sci Rep. 2020; 10(1):1-10.

9. Maher MA, Abdelaziz A, Ellaithy M, Bazeed MF. Prevention of preterm birth: a randomized trial of vaginal compared with intramuscular progesterone. Acta Obstet Gynecol Scand. 2013;92(2):215-22. https://doi.org/10.1111/a ogs.12017.

10. Moroz LA, Simhan HN. Rate of sonographic cervical shortening and the risk of spontaneous preterm birth. A J Obstet Gynecol. 2012;206(3):234. e1-5.

11. Fonseca EB, Celik E, Parra M, Singh M, Nicolaides KH. Progesterone and the risk of preterm birth among women with a short cervix. N Engl J Med. 2007;357(5):462-9. https://doi.org/10.1056/NEJMoa067815.

12. Kuusela $P$, Jacobsson $B$, Söderlund $M$, Bejlum C, Almström $E$, Ladfors $L$, et al. Transvaginal sonographic evaluation of cervical length in the second trimester of asymptomatic singleton pregnancies, and the risk of preterm delivery. Acta Obstet Gynecol Scand. 2015;94(6):598-607. https://doi.org/1 0.1111 /aogs.12622

13. Maerdan M, Shi C, Zhang X, Fan L. The prevalence of short cervix between 20 and 24 weeks of gestation and vaginal progesterone for prolonging of gestation. J Maternal-Fetal Neonatal Med. 2017;30(14):1646-9. https://doi. org/10.1080/14767058.2016.1220528.

14. Sfakianaki AK, Norwitz ER. Mechanisms of progesterone action in inhibiting prematurity. J Maternal-Fetal Neonatal Med. 2006;19(12):763-72. https://doi. org/10.1080/14767050600949829

15. Kuon R-J, Voß P, Rath W. Progesterone for the prevention of preterm birthan update of evidence-based indications. Geburtshilfe Frauenheilkd. 2019; 79(8):844-53. https://doi.org/10.1055/a-0854-6472.

16. Da Fonseca E, Bittar R, Carvalho MH, Zugaib M. Prophylactic administration of progester-one by vaginal suppository to reduce the incidence of spontaneous preterm birth in women at in-creased risk: a randomized placebo-controlled double-blind study. Am J Obstet Gynecol. 2003;188(2): 419-24. https://doi.org/10.1067/mob.2003.41.

17. Dodd JM, Flenady VJ, Cincotta R, Crowther CA. Progesterone for the prevention of preterm birth: a systematic review. Obstet sGynecol. 2008; 112(1):127-34. https://doi.org/10.1097/AOG.0b013e31817d0262.

18. Romero R, Stanczyk FZ. Progesterone is not the same as $17 a-$ hydroxyprogesterone caproate: implications for obstetrical practice. Am J Obstet Gynecol. 2013;208(6):421-6. https://doi.org/10.1016/j.ajog.2013. 04.027.

19. Romero R, Conde-Agudelo A, Da Fonseca E, O'Brien JM, Cetingoz E, Creasy GW, et al. Vaginal progesterone for preventing preterm birth and adverse perinatal outcomes in singleton gestations with a short cervix: a metaanalysis of individual patient data. Am J Obstet Gynecol. 2018;218(2):161-80. https://doi.org/10.1016/j.ajog.2017.11.576.

20. Meis PJ, Klebanoff M, Thom E, Dombrowski MP, Sibai B, Moawad AH, et al. Prevention of recurrent preterm delivery by 17 alpha-hydroxyprogesterone caproate. N Engl J Med. 2003;348(24):2379-85. https://doi.org/10.1056/ NEJMoa035140.

21. Saccone G, Khalifeh A, Elimian A, Bahrami E, Chaman-Ara K, Bahrami M, et al. Vaginal progesterone vs intramuscular 17a-hydroxyprogesterone caproate for prevention of recurrent spontaneous preterm birth in singleton gestations: systematic review and meta-analysis of randomized controlled trials. Ultrasound Obstet Gynecol. 2017:49(3):315-21. https://doi.org/10.1002/ uog. 17245.

22. Shirodkar V. A new method of operative treatment for habitual abortions in the second trimester of pregnancy. Antiseptic. 1955;52:299-300. 
23. de Lignieres B, Dennerstein L, Backstrom T. Influence of route of administration on progesterone metabolism. Maturitas. 1995;21(3):251-7. https://doi.org/10.1016/0378-5122(94)00882-8.

24. Endnote X9. Clarivate analytics. Philadelphia: Thomson Reuters; 2019.

25. Review Manager (RevMan). [Computer program]. Copenhagen The Nordic Cochrane Centre: The Cochrane Collaboration; 2014.

26. Tufanaru CMZ, Aromataris E, Campbell J, Hopp L. Chapter 3: Systematic reviews of effectiveness. In: MZ AE, editor. Joanna Briggs Institute Reviewer's Manual: The Joanna Briggs Institute; 2017.

27. Schünemann H BJ, Guyatt G, Oxman A. Handbook for grading the quality of evidence and the strength of recommendations using the GRADE approach (updated October 2013) GRADE Working Group, 2013. [Available from: guidelinedevelopment.org/app/handbook/handbook.html].

28. Higgins JPT, Thomas J, Chandler J, Cumpston MLT, Page MJ, Welch VA. Chapter 10: Analysing data and undertaking meta-analyses. In: In: Cochrane Handbook for Systematic Reviews of Interventions version 60 (updated July 2019) [Internet]. Cochrane. Available from: www.training.cochrane.org/ha ndbook; 2019. https://doi.org/10.1002/9781119536604.

\section{Publisher's Note}

Springer Nature remains neutral with regard to jurisdictional claims in published maps and institutional affiliations.

Ready to submit your research? Choose BMC and benefit from:

- fast, convenient online submission

- thorough peer review by experienced researchers in your field

- rapid publication on acceptance

- support for research data, including large and complex data types

- gold Open Access which fosters wider collaboration and increased citations

- maximum visibility for your research: over $100 \mathrm{M}$ website views per year

At BMC, research is always in progress.

Learn more biomedcentral.com/submissions 